\title{
NONLINEAR ELECTROHYDRODYNAMIC INSTABILITY OF A JET*
}

\author{
BY \\ R. KANT AND S. K. MALIK \\ Panjab University, Chandigarh
}

\begin{abstract}
A weakly nonlinear theory of an electrohydrodynamic jet held together by capillary forces is investigated. The evolution of two-dimensional wave packets on the surface of the jet is shown to be governed by a nonlinear Schrödinger equation. It is found that the wave train of constant amplitude is unstable against modulation. The instability sets in at higher wave numbers as compared to the case of an unelectrified jet.
\end{abstract}

1. Introduction. The stability of a liquid inviscid jet in the presence of electrostatic forces was investigated by Rayleigh [1]. He showed that electrostatic forces have a destabilizing influence and cause the amplification of nonaxisymmetric perturbations. Melcher [2] and Michael [3] have studied the stability of a circular jet projected along the axis of a cylinder raised to a fixed potential. Yuen [4], Nayfeh [5], and Kakutani et al. [6] reinvestigated this classical problem without an electric field by allowing for the weak nonlinearity of the wave amplitude. The experimental studies of the stability of a liquid jet have shown that Rayleigh's [1] linearized analysis is inadequate to explain the observations. (See Crane et al. [7], Donnelly and Glaberson [8], Chaudhary and Maxworthy [9]). Malik and Singh [10] examined the break-up of an electrohydrodynamic jet, held together in equilibrium by capillary forces. It is shown that the jet breaks into main drops and satellites, whose sizes are sensitive to the applied electric field. Their analysis is valid for wave numbers $k \leqslant k_{c}$, where $k_{c}$ is the linear cutoff wave number. The mechanism of growth of capillary instability of an electrohydrodynamic jet was used in fluid amplifiers by Pierce [11]. These experiments are reviewed in a monograph by Melcher [2]. In all these experiments aperiodic disturbances were observed. With a view to explain these disturbances, we remove the restriction of periodicity for traveling waves and take into account full amplitude modulations.

The study of nonlinear dispersive waves has received considerable attention in recent years (see Whitham [12], Karpman [13]). The aim of this paper is to investigate the

\footnotetext{
* Received July 1, 1981.
} 
asymptotic behavior of weakly nonlinear dispersive waves on the surface of an electrohydrodynamic jet by using the method of multiple scales so as to take into account modulation for progressive waves with wave numbers larger than the cutoff wave number and amplitude modulation of standing waves near the cutoff wave number. The evolution of the finite-amplitude wave packets on the surface of the jet is governed by a nonlinear Schrödinger equation which has a progressive wave train solution in terms of Jacobian elliptic functions. The wave train solution of constant amplitude is unstable against modulation and the instability sets in at a much higher wave number as compared to the case when the electric field is zero. This instability gives the possibility that a wave packet may spontaneously tend to form lumps, sometimes also called envelope solitons. The equation for amplitude modulation for standing waves can be described by a nonlinear Schrödinger equation with the role of time and space variables interchanged. The nonlinear cutoff wave number which separates the region of stability from instability is then obtained from this equation.

2. Formulation. We study two-dimensional finite amplitude wave propagation on the surface of a perfectly conducting, inviscid and incompressible jet of radius $a$. The jet is subjected to an electric field $E_{0}$ at the surface of the undeformed jet. The motion ensues from rest and the flow field generated due to wave motion is assumed to be irrotational and axisymmetric. The basic equations governing velocity and electrostatic potentials are

$$
\begin{gathered}
\nabla^{2} \Omega=0, \quad 0 \leqslant r \leqslant 1+\eta(z, t), \\
\nabla^{2} \phi=0,
\end{gathered}
$$

where $\eta$ denotes the elevation of the free surface measured from the unperturbed level. All the various physical quantities are normalized by introducing dimensionless variables $\omega^{2} \rho a^{3} / T, a K E_{0}^{2} / T$ and $a$. Here $\rho, K$, and $T$ are density, the dielectric constant, and the surface tension of the fluid, respectively. The boundary conditions at the free surface $r=1+\eta(z, t)$ are

$$
\begin{gathered}
\frac{\partial \eta}{\partial t}+\frac{\partial \Omega}{\partial r}=\frac{\partial \Omega}{\partial z} \frac{\partial \eta}{\partial z} \\
\phi=\text { constant } \\
\frac{\partial \Omega}{\partial t}-\frac{1}{2}\left(\left(\frac{\partial \Omega}{\partial r}\right)^{2}+\left(\frac{\partial \Omega}{\partial z}\right)^{2}\right)+\frac{\partial^{2} \eta}{\partial z^{2}}\left(1+\left(\frac{\partial \eta}{\partial z}\right)^{2}\right)^{-3 / 2} \\
-(1+\eta)^{-1}\left(1+\left(\frac{\partial \eta}{\partial z}\right)^{2}\right)^{-1 / 2}+1+\frac{\alpha}{2}\left(\frac{\partial \phi}{\partial \hat{n}}\right)^{2}=\text { constant }
\end{gathered}
$$

where

$$
\alpha=K E_{0}^{2} / 4 \pi T,
$$

and $\hat{n}$ represents the outward-drawn normal.

3. Perturbation scheme. To investigate the modulation of a weakly nonlinear quasimonochromatic wave with narrow bandwidth, we employ the derivative expansion method. 
We now introduce the variables

$$
Z_{n}=\varepsilon^{n} Z, \quad t_{n}=\varepsilon^{n} t \quad(n=0,1,2, \ldots, N),
$$

where $\varepsilon$ represents the strength of the nonlinearity. In order to describe the nonlinear interactions of small but finite amplitude of the waves, we expand $\eta, \Omega$, and $\phi$ in the following asymptotic series:

$$
\begin{aligned}
\eta(Z, t) & =\sum_{n=1}^{N+1} \varepsilon^{n} \eta_{n}\left(Z_{0}, Z_{1}, \ldots, Z_{n} ; t_{0}, t_{1}, \ldots, t_{n}\right)+O\left(\varepsilon^{N+2}\right), \\
\Omega(Z, t, r) & =\sum_{n=1}^{N+1} \varepsilon^{n} \Omega_{n}\left(Z_{0}, Z_{1}, \ldots, Z_{n} ; t_{0}, t_{1}, \ldots, t_{n}, r\right)+O\left(\varepsilon^{N+2}\right), \\
\phi(Z, t, r) & =\sum_{n=0}^{N+1} \varepsilon^{n} \phi_{n}\left(Z_{0}, Z_{1}, \ldots, Z_{n} ; t_{0}, t_{1}, \ldots, t_{n}, r\right)+O\left(\varepsilon^{N+2}\right), \\
\frac{\partial}{\partial t} & =\sum_{n=0}^{N} \varepsilon^{n} \frac{\partial}{\partial t_{n}}+O\left(\varepsilon^{N+1}\right)
\end{aligned}
$$

and

$$
\frac{\partial}{\partial x}=\sum_{n=0}^{N} \varepsilon^{n} \frac{\partial}{\partial x_{n}}+O\left(\varepsilon^{N+1}\right) .
$$

For the problem under investigation, it is sufficient to take $N=2$ as far as the lowest significant interactions are concerned. On employing Taylor's expansion around the undisturbed surface $r=1$ for boundary conditions (3)-(4), use of Eqs. (6)-(11) lead to linear and successive nonlinear partial differential equations of various orders (see Appendix).

\section{Linear theory and second-order solutions.}

4a) Linear theory. The progressive wave solutions of equations of the first-order problem given by Eqs. (A.6) to (A.8) yield

$$
\begin{aligned}
& \eta_{1}=(A \exp (i \theta)+\bar{A} \exp (-i \theta)), \\
& \Omega_{1}=i \omega k^{-1} \frac{I_{0}(k r)}{I_{1}(k)}(A \exp (i \theta)-\bar{A} \exp (-i \theta))+B_{1}, \\
& \phi_{1}=\frac{K_{0}(k r)}{K_{0}(k)}(A \exp (i \theta)+\bar{A} \exp (-i \theta)),
\end{aligned}
$$

where

$$
\theta=k Z_{0}-\omega t_{0} .
$$

The amplitude $A$ is a function of the slower variables $Z_{1}, Z_{2} ; t_{1}, t_{2}$ and $B_{1}$ is assumed to be a real constant and is independent of lower scales $Z_{0}, t_{0}$. For the above solutions to be nontrivial the frequency $\omega$ and the wave number $k$ must satisfy the dispersion relation

$$
D(\omega, k)=\omega^{2} \beta(k)+k\left[\left(1-k^{2}\right)-\alpha(1-k \gamma(k))\right]=0,
$$


where

$$
\beta(k)=I_{0}(k) / I_{0}(k) \text { and } \gamma(k)=K_{1}(k) / K_{0}(k) .
$$

For long wavelengths (i.e., $k \rightarrow 0$ ), the above result reduces to Rayleigh's result. The jet is unstable for all perturbations which have wave number less than $k_{c}$, where $k_{c}$ is determined by the transcendental equation

$$
1-k^{2}-\alpha(1-k \gamma(k))=0
$$

In Figure 1, we have shown the variation of the linear cutoff wave number $k_{c}$ as a function of $\alpha$. The value of $k_{c}$ increases with increase in $\alpha$.

Traveling wave solutions are possible for $k \geqslant k_{c}$. Since our aim is to investigate the amplitude modulation of progressive waves, we now proceed to the second and third-order problems.

4b) Second-order solutions. We now substitute the traveling wave solutions given by Eqs. (12)-(14) into the right-hand side of Eqs. (A.9)-(A.12) and get

$$
\begin{gathered}
L\left[\Omega_{2}\right]=2 \omega \frac{I_{0}(k r)}{I_{1}(k)} \frac{\partial A}{\partial z_{1}} \exp (i \theta)+\text { c.c., } \\
L\left[\phi_{2}\right]=-2 i k \frac{K_{0}(k r)}{K_{0}(k)} \frac{\partial A}{\partial Z_{1}} \exp (i \theta)+\text { c.c., } \\
M\left[\eta_{2}, \Omega_{2}\right]=-\frac{\partial A}{\partial t_{1}} \exp (i \theta)+i \omega(1-2 k \beta(k)) A^{2} e^{2 i \theta}+\text { c.c., at } r=1 \\
N\left[\eta_{2}, \Omega_{2}, \phi_{2}\right]=-i\left(\omega k^{-1} \beta(k) \frac{\partial A}{\partial t_{1}}+2 k \frac{\partial A}{\partial Z_{1}}\right) \exp (i \theta) \\
+\frac{1}{2}\left[\omega^{2}\left(\beta^{2}(k)-3\right)+2+k^{2}-\alpha\left\{k^{2} \gamma^{2}(k)-2 k \gamma(k)\right.\right. \\
+\left[\omega^{2}\left(\beta^{2}(k)-1\right)+2-k^{2}\left(1+\alpha\left\{k^{-1} \gamma(k)-k^{2}+3\right)\right\}\right] A^{2} \exp (2 i \theta)+\text { c.c. } \\
+\gamma^{2}(k)-2 k \gamma(k)-2 k^{2}\left(1+k^{-1} \gamma(k)\right)
\end{gathered}
$$

and

$$
O\left[\eta_{2}, \phi_{2}\right]=(2 k \gamma(k)-1)\left[\frac{A^{2}}{2} \exp (2 i \theta)+A \vec{A}\right] \quad \text { at } r=1
$$

The second-order problem (19)-(23) has a uniformly valid solution if and only if the inhomogeneous part is orthogonal to every solution of the corresponding adjoint homogeneous part. This leads to the nonsecularity condition

$$
2 \omega \beta(k) \frac{\partial A}{\partial t_{1}}-\left[\omega^{2} \beta(k)+1-3 k^{2}-\alpha\left\{1-k\left(2 \gamma(k)+k \gamma^{\prime}(k)\right)\right\}\right] \frac{\partial A}{\partial Z_{1}}=0 .
$$

Equivalently we have

$$
\frac{\partial A}{\partial t_{1}}+V_{g} \frac{\partial A}{\partial Z_{1}}=0
$$




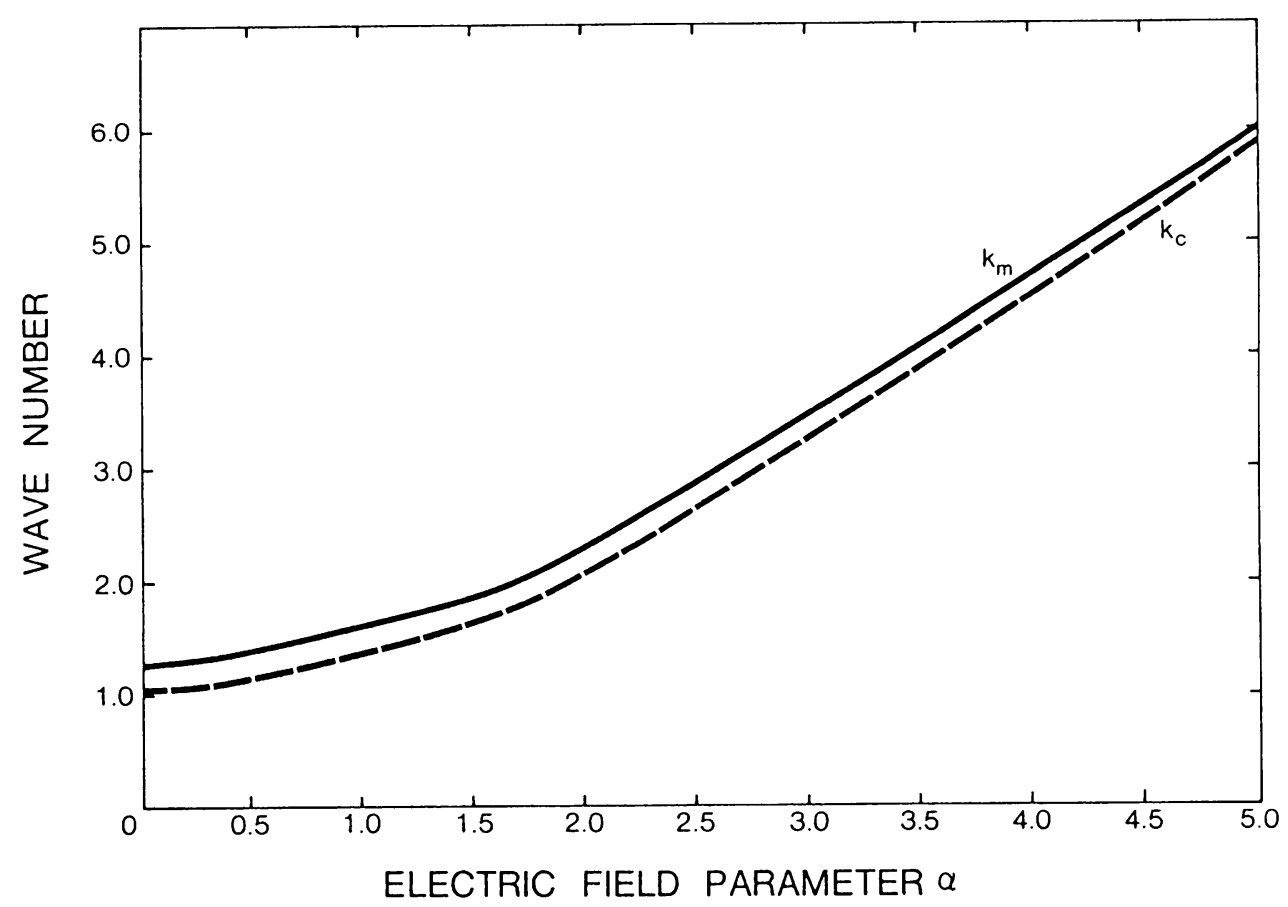

FIG. 1. The variation of the cutoff wave numbers $k_{c}$ and $k_{M}$ with respect to $\alpha$.

together with its complex conjugate relation. Here $V_{g}=d \omega / d k$ represents the group velocity. Eq. (24) implies that the amplitude $A$ is a constant in a frame of reference moving with the group velocity of the waves. With this solvability condition the uniformly valid solutions of the second-order problem are

$$
\begin{aligned}
\eta_{2}= & -i \omega^{-1}\left(\frac{\partial A}{\partial t_{1}}+\omega \beta(k) \frac{\partial A}{\partial Z_{1}}\right) \exp (i \theta) \\
& +q_{2} A^{2} \exp (2 i \theta)+\text { c.c. }-(1-\alpha)^{-1}\left(2 q_{1} A \bar{A}-\frac{\partial B_{1}}{\partial t_{1}}\right), \\
\Omega_{2}= & {\left[\omega k^{-1} r \frac{I_{1}(k r)}{I_{1}(k)} \frac{\partial A}{\partial Z_{1}}\right] \exp (i \theta) } \\
& +i q_{3} A^{2} \exp (2 i \theta) \frac{I_{0}(2 k r)}{I_{1}(2 k)}+\text { c.c. }+B_{2}, \\
\phi_{2}= & {\left[i r \frac{K_{1}(k r)}{K_{0}(k)} \frac{\partial A}{\partial Z_{1}}\right] \exp (i \theta) } \\
& +q_{4} \frac{K_{0}(2 k r)}{K_{0}(2 k)} A^{2} \exp 2 i \theta+\text { c.c. }+B_{3},
\end{aligned}
$$


where

$$
\begin{aligned}
q_{1}= & \frac{1}{2}\left[\omega^{2}\left(\beta^{2}(k)-1\right)+2-k^{2}-\alpha\left\{k^{2}\left(\gamma^{2}(k)-1\right)+3-4 k \gamma(k)\right\}\right], \\
q_{2}= & \frac{k}{D(2 \omega, 2 k)}\left[\omega^{2}\left(\beta^{2}(k)-3-2 k^{-1} \beta(2 k)(1-2 k \beta(k))\right)+2+k^{2}\right. \\
& \left.-\alpha\left\{k^{2} \gamma^{2}(k)-4 k \gamma(k)-3 k^{2}+3+2 k \gamma(2 k)(2 k \gamma(2 k)-1)\right\}\right], \\
q_{3}= & \frac{\omega}{2 k}\left[2 q_{2}+1-2 k \beta(k)\right], \\
q_{4}= & q_{2}+\left(k \gamma(k)-\frac{1}{2}\right),
\end{aligned}
$$

and

$$
D(2 \omega, 2 k)=4 \omega^{2} \beta(2 k)+2 k\left[\left(1-4 k^{2}\right)-\alpha(1-2 k \gamma(2 k))\right] .
$$

Here $B_{2}$ and $B_{3}$ are arbitrary constants. Furthermore, we assume that $D(2 \omega, 2 k) \neq 0$. The case $D(2 \omega, 2 k)=0$ corresponds to the second harmonic resonance.

5. Amplitude modulation of traveling waves for $k>k_{c}$. In order to obtain the amplitude modulation for the traveling wave, we look into a third-order problem given by Eqs. (A.13)-(A.17). After some straightforward manipulations, the constant terms give the following nonsecularity condition for $\eta_{3}$ :

$$
\partial B_{1} / \partial Z_{1}=q_{5} A \bar{A}+C\left(Z_{2}, t_{2}\right),
$$

where

$$
q_{5}=\left(\alpha^{*} V_{g}^{2}+\frac{1}{2}\right)^{-1}\left[2 \omega \beta(k)-\left(2 q_{1} \alpha^{*}+1\right) V_{g}\right],
$$

with $\alpha^{*}=(1-\alpha)^{-1}$, and $C\left(Z_{2}, t_{2}\right)$ is a constant of integration to be determined by imposing suitable initial or boundary conditions. We impose the condition that there is no steady flow at infinity in the space of variable $Z_{1}$, implying that

$$
\text { ว } B_{1} / \partial Z_{1}=0, \quad A=A_{0} \quad \text { as } Z_{1} \rightarrow-\infty .
$$

This condition gives $C\left(Z_{2}, t_{2}\right)=-q_{5} A_{0} \overline{A_{0}}$.

Equation (33) represents the induced steady flow along the axis of the jet due to nonlinearity. On using Eqs. (12)-(17) and (25)-(36) into third-order perturbation Eqs. (A.13)-(A.17), we get the following nonsecularity condition:

$$
i\left(\frac{\partial A}{\partial t_{2}}+V_{g} \frac{\partial A}{\partial Z_{2}}\right)+P \frac{\partial^{2} A}{\partial Z_{1}^{2}}=Q A^{2} \bar{A}+R A,
$$

where

$$
\begin{aligned}
& P=\frac{1}{2} \frac{d V_{g}}{d k} \\
& Q=\left(\omega k^{-1} \beta(k) R_{1}+R_{2}+\alpha k \gamma(k) R_{3}\right) k\left(\frac{\partial D}{\partial \omega}\right)^{-1} \\
& R=\left(T_{1} \omega k^{-1} \beta(k)+T_{2}\right) C V_{g} K\left(\frac{\partial D}{\partial \omega}\right)^{-1}
\end{aligned}
$$


where

$$
\begin{gathered}
R_{1}=2 k q_{3}(k \beta(2 k)-1)+\omega k \beta(k)\left(q_{2}+d-\frac{1}{2}\right)+\omega\left(d+q_{2}+\frac{3}{2} k^{2}+1\right), \\
R_{2}=2 \omega k q_{3}(\beta(k) \beta(2 k)-1)+\omega^{2}\left(\frac{5}{2} k \beta(k)+\frac{1}{2}-d-q_{2}\right) \\
-\omega \beta(k) q_{5}-k^{2}-3+2\left(q_{2}+d-k q_{2}\right) \\
-\alpha\left[2 k q_{4}(2 k \gamma(2 k) \gamma(k)-k-2 \gamma(2 k))-\left(d+q_{2}\right)(k(k+2 \gamma(k))-1)\right. \\
\left.\quad-k^{2} \gamma(k)\left(\frac{11}{2}+3 \gamma(k)\right)-\frac{5 k^{2}}{2}-6\right], \\
R_{3}=(k \gamma(k)-1)\left(q_{2}+d\right)+2 k \gamma(2 k) q_{4}-\frac{3}{2} k(1+k \gamma(k))+1, \\
T_{1}=\omega \alpha^{*} V_{g}(k \beta(k)-1)+k, \\
T_{2}=\alpha \alpha^{*}[k(k+2 \gamma(k))+1],
\end{gathered}
$$

and

$$
d=\alpha^{*}\left[2 q_{1}+V_{g} q_{5}\right]
$$

Introducing the transformations $\xi=\varepsilon^{-1}\left(Z_{2}-V_{g} t_{2}\right)=Z_{1}-V_{g} t_{1} ; \tau=\varepsilon^{2} t_{1}$, and

$$
A=A \exp \left(-i \int^{\tau} R\left(\tau^{\prime}\right) d \tau^{\prime}\right)
$$

the nonlinear Schrödinger equation takes the form

$$
i \frac{\partial A}{\partial \tau}+P \frac{\partial^{2} A}{\partial \xi^{2}}=Q A^{2} \bar{A}
$$

The equilibrium solution of this equation represents a wave train which can be expressed in terms of Jacobian elliptic functions. The solitary waves, phase jump, and the progressive wave train of constant amplitude are special cases. (see Kakutani et al. [6], Kant et al. [14]). For the plane wave one can show that the band width of the spectrum is $O\left(\varepsilon^{2}\right)$ in wave number space and $O(\varepsilon)$ in the frequency space for progressive waves. Also, such a solution is unstable against modulation if $P Q<0$. This criterion, for $\alpha=0$, is satisfied for the wave numbers $k>1.28$. The plot of the wave number $k_{M}$ at which the modulational instability sets in as a function of $\alpha$ is shown in Fig. 1. It is interesting to note that this instability sets in at much higher wave numbers, when the external electric field is applied to the jet.

The physical mechanism of modulational instability can be described by using the wave kinetics approach due to Whitham [12]. For nonlinear waves, the phase velocity of the wave is proportional to its amplitude. If the waves are subjected to a long wavelength perturbation, then for $Q>0$, the waves falling in the crest of the perturbation will move faster than the rest. This will lead to compression and energy concentration in front of the crest, thereby leading to an increase in the local wave number in this region. The group velocity represents the velocity with which energy is transported into the medium. Therefore, for $P<0$, the energy concentrated in front of the crest will be preferentially deposited on the crest because of the wave number dependence of the group velocity, and 
this will lead to an instability. On the other hand, for $P>0$, the energy will be transported into the depleted region behind the crest. Similarly for $Q<0$, the instability occurs when $P>0$.

The initial value problem for Eq. (47) can also be solved exactly by using an inverse scattering technique (see Zakharov and Shabat [15]). It is found that if $P Q>0$, any initial disturbance for $A$ will disperse out, but if $P Q<0$, soliton envelopes will emerge.

6. Amplitude modulation of standing waves. We now discuss the waves near $k=k_{c}$ and $\omega=0$. The carrier wave is not a traveling wave but instead a standing wave. For this case the coefficients $P$ and $Q$ become singular as $k$ approaches $k_{c}$. The previous analysis needs modification.

The starting solutions of the first-order problem which we take here are:

$$
\begin{gathered}
\eta_{1}=A\left(Z_{1}, Z_{2} ; t_{1}, t_{2}\right) \exp \left(i k_{c} Z\right)+\text { c.c. } \\
\Omega_{1}=B_{1}\left(Z_{1}, Z_{2} ; t_{1}, t_{2}\right),
\end{gathered}
$$

and

$$
\phi_{1}=\left(A \exp \left(i k_{c} Z\right)+\text { c.c. }\right) \frac{K_{0}\left(k_{c} r\right)}{K_{0}\left(k_{c}\right)} .
$$

Proceeding as before, we find the nonsecularity condition for the second-order perturbation

$$
\partial A / \partial Z_{1}=0,
$$

which implies that the amplitude $A\left(Z_{1}, Z_{2}, t_{1}, t_{2}\right)$ is independent of the slower space variable $Z_{1}$. The uniformly valid solution of the second-order problem is:

$$
\begin{aligned}
& \eta_{2}=\alpha^{*}\left(s_{1} A \bar{A}-\frac{\partial B_{1}}{\partial t_{1}}\right)+s_{2} A^{2} \exp (2 i \theta)+\text { c.c. } \\
& \Omega_{2}=-\frac{I_{0}\left(k_{c} r\right)}{k_{c} I_{1}\left(k_{c}\right)} \frac{\partial A}{\partial t_{1}} \exp (i \theta)+\text { c.c. }+B_{4}, \\
& \phi_{2}=-i r \frac{K_{1}\left(k_{c} r\right)}{K_{0}\left(k_{c}\right)} \frac{\partial A}{\partial Z_{1}} \exp (i \theta)+s_{3} \frac{K_{0}\left(2 k_{c} r\right)}{K_{0}\left(2 k_{c}\right)} A^{2} \exp (2 i \theta)+\text { c.c. }+B_{5},
\end{aligned}
$$

where

$$
\begin{aligned}
s_{1}= & 2-k_{c}^{2}-\alpha\left[k_{c}^{2} \gamma^{2}\left(k_{c}\right)-2 k_{c} \gamma\left(k_{c}\right)-2 k_{c}^{2}\left(1+k_{c}^{-1} \gamma\left(k_{c}\right)\right)+k_{c}^{2}+3\right], \\
s_{2}= & \frac{1}{2\left[1-4 k_{c}^{2}+\alpha\left(1-2 k_{c} \gamma\left(2 k_{c}\right)\right)\right]}\left[2+k_{c}^{2}-\alpha\left\{k_{c}^{2} \gamma^{2}\left(k_{c}\right)\right.\right. \\
& \left.\left.-2 k_{c} \gamma\left(k_{c}\right)-2 k_{c}\left(k_{c}+\gamma\left(k_{c}\right)\right)-k_{c}^{2}+3+2 k_{c} \gamma\left(2 k_{c}\right)\left(2 k_{c} \gamma\left(2 k_{c}\right)-1\right)\right\}\right], \\
s_{3}= & s_{2}+\left(k_{c} \gamma\left(k_{c}\right)-\frac{1}{2}\right) .
\end{aligned}
$$

Considering only the constant terms in the third-order problem, we get the following nonsecularity condition:

$$
\partial B_{1} / \partial t_{1}=\left(s_{1}+\left(\alpha^{*}\right)^{-1}\right) A \bar{A}+C\left(Z_{2}, t_{2}\right),
$$


where $C\left(Z_{2}, t_{2}\right)$ is an integration constant which may be determined by initial or boundary conditions. On invoking the boundary condition that there is no flow at infinity, we get

$$
C\left(Z_{2}, t_{2}\right)=-\left(s_{1}+\left(\alpha^{*}\right)^{-1}\right) A_{0} \overline{A_{0}} .
$$

Proceeding as before, after some straightforward manipulations, the nonsecular condition for the third-order perturbation is given by

where

$$
i \frac{\partial A}{\partial Z_{2}}+\check{P} \frac{\partial^{2} A}{\partial t_{1}^{2}}=\check{Q} A^{2} \bar{A}+\check{R} A,
$$

$$
\begin{aligned}
& \check{P}=\frac{-\beta\left(k_{c}\right)}{k_{c}^{2}\left[2-\alpha\left(\gamma^{2}\left(k_{c}\right)-1\right)\right]}, \\
& \check{Q}=\frac{1}{k_{c}\left[2-\alpha\left(\gamma^{2}\left(k_{c}\right)-1\right)\right]}\left[-\frac{3 k_{c}^{4}}{2}+\frac{k_{c}^{2}}{2}-5+2 s_{2}\left(1-k_{c}^{2}\right)-\alpha \tilde{R}_{2}-\alpha k_{c} \gamma\left(k_{c}\right) \tilde{R}_{3}\right],
\end{aligned}
$$

$$
\begin{aligned}
\check{R} & =-\frac{k_{c} T_{2} c}{\left[2-\alpha\left(\gamma^{2}\left(k_{c}\right)-1\right)\right]}, \\
\tilde{R}_{2} & =\left[2 k_{c} s_{3}\left\{k_{c} \gamma\left(2 k_{c}\right) \gamma\left(k_{c}\right)-k_{c}-2 \gamma\left(k_{c}\right)\right\}-\left(s_{2}-1\right)\left\{k_{c}\left(k_{c}+2 \gamma\left(k_{c}\right)\right)+1\right\}\right. \\
& \left.-k_{c}^{3} \gamma\left(k_{c}\right)+3 k_{c} \gamma\left(k_{c}\right)\left\{-k_{c}\left(\frac{k_{c}}{2}+\gamma\left(k_{c}\right)\right)+3\right\}+\frac{3 k_{c}^{2}}{2}-2\right],
\end{aligned}
$$

and

$$
\tilde{R}_{3}=\left(k_{c} \gamma\left(k_{c}\right)-1\right)\left(s_{2}-1\right)+2 k_{c} \gamma\left(2 k_{c}\right) s_{3}-\frac{3}{2} k_{c}\left\{1+k_{c} \gamma\left(k_{c}\right)\right\}+1 .
$$

The equation above is the nonlinear Schrödinger equation with the role of space and time variables interchanged. The interaction term $\breve{R} A$ can be eliminated by the transformation

$$
A\left(Z_{2}, t_{1}\right)=A \exp \left(-i \int^{Z_{2}} \check{R}\left(Z_{2}^{\prime}\right) d Z_{2}^{\prime}\right) .
$$

The equilibrium solutions of (59) can be expressed in terms of the Jacobian elliptic functions. A solitary wave, the phase jump, and the wave train of constant amplitude can be obtained as special cases.

We now consider the wave train solution of the form

$$
A\left(Z_{2}, t_{1}\right)=a_{0} \exp \left(i\left(K Z_{2}-\Omega t_{1}\right)\right),
$$

where $a_{0}$ is a constant. Substituting we get the dispersion equation

$$
\Omega^{2}=-\frac{1}{\ddot{P}}\left(K+\check{Q}\left|a_{0}\right|^{2}\right) .
$$

For $\Omega$ to be imaginary, we require $K<-\check{Q}\left|a_{0}\right|^{2}$ if $\check{P}<0$. Thus the nonlinear cutoff wave number is

$$
k_{n}=k_{c}\left[1+Q_{1}\left|a_{0}\right|^{2} \varepsilon^{2}\right]-\frac{\varepsilon^{2}}{Z_{2}} \int^{Z_{2}} \tilde{R}\left(Z_{2}^{\prime}\right) d Z_{2}^{\prime},
$$


where

$$
Q_{1}=-\check{Q},
$$

and $k_{c}$. represents the cutoff wave number predicted by the linear theory. The above relation reveals that the cutoff wave number $k_{n}$ depends upon the initial conditions and is amplitude dependent. We can recover the results of Kakutani et al. [6] on letting $\alpha=0$ and $k_{c}=1$. Furthermore, the nonlinearity changes the range of unstable wave numbers within the jet, and the bandwidth of the spectrum is of the order $O\left(\varepsilon^{2}\right)$ in the wave number space for the standing waves. We observe from Fig. 2 that the value of $Q_{1}$ first increases, then goes to zero and further decreases with the increase of $\alpha$. The function $Q_{1}$ is zero at $\alpha=1.447$. At this value of $\alpha$ the nonlinearity has no effect on the critical wave number.

7. Discussion. We have shown in this paper that the wave train solution of constant amplitude with greater wave number than the linear cutoff wave number $k_{c}$ is unstable against modulation on the surface of an electrohydrodynamic jet. The wave number $k_{M}$ at which the modulational instability sets in increases with the increase of the applied electric field. This instability will ultimately result in the appearence of a series of solitons (see Zakharov and Shabat [15]). It is also demonstrated that there exists a critical value of $\alpha$ at which the nonlinearity has no effect on the critical wave number.

We are thankful to Professor M. Singh for his interest in this work.

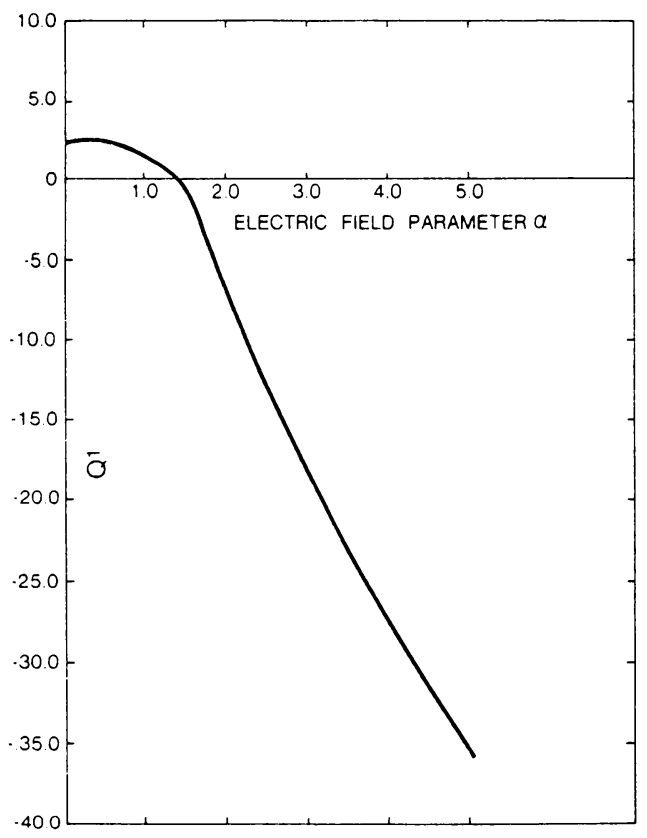

FIG. 2. The variation of $Q_{1}$ with the electric field parameter $\alpha$. 
Appendix. We introduce the linear operators defined as

$$
\begin{aligned}
\mathbf{L} & =\frac{\partial^{2}}{\partial r^{2}}+\frac{1}{r} \frac{\partial}{\partial r}+\frac{\partial^{2}}{\partial Z^{2}}, \\
\mathbf{M}\left[\eta_{1}, \Omega_{1}\right] & =\frac{\partial \eta_{1}}{\partial t_{0}}+\frac{\partial \Omega_{1}}{\partial r}, \\
\mathbf{N}\left[\eta_{1}, \Omega_{1}, \phi\right] & =\frac{\partial \Omega_{1}}{\partial t_{0}}+\frac{\partial^{2} \eta_{1}}{\partial Z_{0}^{2}}+\eta_{1}+\alpha \frac{\partial \phi_{0}}{\partial r}\left(\frac{\partial \phi_{1}}{\partial r}+\eta_{1} \frac{\partial^{2} \phi_{0}}{\partial r^{2}}\right), \\
\mathbf{O}\left[\eta_{1}, \phi_{1}\right] & =\phi_{1}+\eta_{1} \frac{\partial \phi_{0}}{\partial r} .
\end{aligned}
$$

The zero-order solutions are given by

$$
\Omega_{0}=0, \quad \phi_{0}=-\ln (r) .
$$

The first-order equations to $O(\varepsilon)$ are:

with the boundary conditions at $r=1$ as

$$
\mathbf{L}\left[\Omega_{1}\right]=0, \quad L\left[\phi_{1}\right]=0
$$

$$
\begin{aligned}
& \mathbf{M}\left[\eta_{1}, \Omega_{1}\right]=0, \quad \mathbf{N}\left[\eta_{1}, \Omega_{1}, \phi_{1}\right]=0, \\
& \mathbf{O}\left[\eta_{1}, \phi_{1}\right]=0 .
\end{aligned}
$$

The second-order problem for $O\left(\varepsilon^{2}\right)$ is given by

$$
\mathbf{L}\left[\Omega_{2}\right]=-\frac{2 \partial^{2} \Omega_{1}}{\partial Z_{0} \partial Z_{1}}, \quad \mathbf{L}\left[\phi_{2}\right]=-2 \frac{\partial^{2} \phi_{1}}{\partial Z_{0} \partial Z_{1}},
$$

with

$$
\begin{aligned}
\mathbf{M}\left[\eta_{2}, \Omega_{2}\right]= & -\frac{\partial \eta_{1}}{\partial t_{1}}-\eta_{1} \frac{\partial^{2} \Omega_{1}}{\partial r^{2}}+\frac{\partial \Omega_{1}}{\partial Z_{0}} \frac{\partial \eta_{1}}{\partial Z_{0}} \quad \text { at } r=1 \\
\mathbf{O}\left[\eta_{2}, \phi_{2}\right]= & -\eta_{1} \frac{\partial \phi_{1}}{\partial r}-\frac{\eta_{1}^{2}}{2} \frac{\partial^{2} \phi_{0}}{\partial r^{2}} \quad \text { at } r=1 \\
\mathbf{N}\left[\eta_{2}, \Omega_{2}, \phi_{2}\right]= & -\frac{\partial \Omega_{1}}{\partial t_{1}}-\eta_{1} \frac{\partial^{2} \Omega_{1}}{\partial t_{0} \partial r}+\frac{1}{2}\left[\left(\frac{\partial \Omega_{1}}{\partial r}\right)^{2}+\left(\frac{\partial \Omega_{1}}{\partial Z_{0}}\right)^{2}\right] \\
& -2 \frac{\partial^{2} \eta_{1}}{\partial Z_{0} \partial Z_{1}}+\eta_{1}^{2}-\frac{1}{2}\left(\frac{\partial \eta_{1}}{\partial Z_{0}}\right)^{2}-\frac{\alpha}{2}\left[\left(\frac{\partial \phi_{1}}{\partial r}\right)^{2}+2 \eta_{1}\left(\frac{\partial \phi_{1}}{\partial r}\right)^{\frac{\partial^{2} \phi_{0}}{\partial r^{2}}}\right. \\
& \left.-2 \frac{\partial \phi_{0}}{\partial r} \frac{\partial \eta_{1}}{\partial Z_{0}} \frac{\partial \phi_{1}}{\partial Z_{0}}+\eta_{1}^{2}\left\{\left(\frac{\partial^{2} \phi_{0}}{\partial r^{2}}\right)^{2}+\frac{\partial \phi_{0}}{\partial r} \frac{\partial^{3} \phi_{0}}{\partial r^{3}}\right\}-\left(\frac{\partial \phi_{0}}{\partial r}\right)^{2}\left(\frac{\partial \eta_{1}}{\partial Z_{0}}\right)^{2}\right]
\end{aligned}
$$

The third-order problem for $O\left(\varepsilon^{3}\right)$ is

$$
\begin{aligned}
& \mathbf{L}\left[\Omega_{3}\right]=-2 \frac{\partial^{2} \Omega_{2}}{\partial Z_{0} \partial Z_{1}}-\frac{\partial^{2} \Omega_{1}}{\partial Z_{2}}-2 \frac{\partial^{2} \Omega_{1}}{\partial Z_{0} \partial Z_{2}}, \\
& \mathbf{L}\left[\phi_{3}\right]=-2 \frac{\partial^{2} \phi_{2}}{\partial Z_{0} \partial Z_{1}}-\frac{\partial^{2} \phi_{1}}{\partial Z_{1}^{2}}-2 \frac{\partial^{2} \phi_{1}}{\partial Z_{0} \partial Z_{2}}
\end{aligned}
$$


with the boundary conditions at $r=1$ being

$$
\begin{aligned}
& \mathbf{M}\left[\eta_{3}, \Omega_{3}\right]=-\frac{\partial \eta_{2}}{\partial t_{1}}-\frac{\partial \eta_{1}}{\partial t_{2}}-\eta_{1} \frac{\partial^{2} \Omega_{2}}{\partial r^{2}}-\eta_{2} \frac{\partial^{2} \Omega_{1}}{\partial r^{2}}-\frac{1}{2} \eta_{1}^{2} \frac{\partial^{3} \Omega_{1}}{\partial r^{3}} \\
& +\frac{\partial \Omega_{1}}{\partial Z_{0}}\left(\frac{\partial \eta_{1}}{\partial Z_{1}}+\frac{\partial \eta_{2}}{\partial Z_{0}}\right)+\frac{\partial \eta_{1}}{\partial Z_{0}}\left(\frac{\partial \Omega_{2}}{\partial Z_{0}}+\frac{\partial \Omega_{1}}{\partial Z_{1}}+\eta_{1} \frac{\partial^{2} \Omega_{1}}{\partial Z_{0} \partial r}\right), \\
& \mathbf{N}\left[\eta_{3}, \Omega_{3}, \phi_{3}\right]=-\frac{\partial \Omega_{2}}{\partial t_{1}}-\frac{\partial \Omega_{1}}{\partial t_{2}}-\eta_{1}\left(\frac{\partial^{2} \Omega_{2}}{\partial t_{1} \partial r}+\frac{\partial^{2} \Omega_{1}}{\partial t_{1} \partial r}\right)-\frac{1}{2} \eta_{1}^{2} \frac{\partial^{3} \Omega_{1}}{\partial t_{0} \partial r^{2}} \\
& -\eta_{2} \frac{\partial^{2} \Omega_{1}}{\partial t_{1} \partial r}+\frac{\partial \Omega_{1}}{\partial r}\left(\frac{\partial \Omega_{2}}{\partial r}+\eta_{1} \frac{\partial^{2} \Omega_{1}}{\partial r^{2}}\right) \\
& +\frac{\partial \Omega_{1}}{\partial Z_{0}}\left(\frac{\partial \Omega_{2}}{\partial Z_{0}}+\eta_{1} \frac{\partial^{2} \Omega_{1}}{\partial Z_{0} \partial r}+\frac{\partial \Omega_{1}}{\partial Z_{1}}\right) \\
& -2 \frac{\partial^{2} \eta_{2}}{\partial Z_{0} \partial Z_{1}}-\frac{\partial^{2} \eta_{1}}{\partial Z_{1}^{2}}-2 \frac{\partial^{2} \eta_{1}}{\partial Z_{0} \partial Z_{2}}+\frac{3}{2}\left(\frac{\partial \eta_{1}}{\partial Z_{0}}\right)^{2} \frac{\partial^{2} \eta_{1}}{\partial Z_{0}^{2}}-\eta_{1}^{3}+2 \eta_{1} \eta_{2} \\
& +\frac{1}{2} \eta_{1}\left(\frac{\partial \eta_{1}}{\partial Z_{0}}\right)^{2}-\frac{\partial \eta_{1}}{\partial Z_{0}}\left(\frac{\partial \eta_{1}}{\partial Z_{1}}+\frac{\partial \eta_{2}}{\partial Z_{0}}\right)-\alpha \frac{\partial \phi_{1}}{\partial r} \cdot \frac{\partial \phi_{2}}{\partial r} \\
& -\alpha \eta_{1}\left(\frac{\partial \phi_{0}}{\partial r} \frac{\partial^{2} \phi_{2}}{\partial r^{2}}+\frac{\partial \phi_{2}}{\partial r} \frac{\partial^{2} \phi_{0}}{\partial r^{2}}+\frac{\partial \phi_{1}}{\partial r} \frac{\partial^{2} \phi_{1}}{\partial r^{2}}\right)-\alpha \eta_{2}\left(\frac{\partial \phi_{0}}{\partial r} \frac{\partial^{2} \phi_{1}}{\partial r^{2}}\right) \\
& -\alpha \eta_{2} \frac{\partial \phi_{1}}{\partial r} \cdot \frac{\partial^{2} \phi_{0}}{\partial r^{2}}-\eta_{1} \eta_{2}\left[\left(\frac{\partial^{2} \phi_{0}}{\partial r^{2}}\right)^{2}+\frac{\partial \phi_{0}}{\partial r} \frac{\partial^{3} \phi_{0}}{\partial r^{3}}\right] \\
& +\alpha \frac{\partial \phi_{0}}{\partial r} \cdot \frac{\partial \eta_{2}}{\partial Z_{0}} \cdot \frac{\partial \phi_{1}}{\partial Z_{0}} \\
& -\frac{\alpha}{2} \eta_{1}^{2}\left(2 \frac{\partial^{2} \phi_{1}}{\partial r^{2}} \frac{\partial^{2} \phi_{0}}{\partial r^{2}}+\frac{\partial \phi_{1}}{\partial r} \frac{\partial^{3} \phi_{0}}{\partial r^{3}}+\frac{\partial \phi_{0}}{\partial r} \frac{\partial^{3} \phi_{1}}{\partial r^{3}}\right) \\
& +\alpha \frac{\partial \phi_{0}}{\partial r} \frac{\partial \eta_{1}}{\partial Z_{1}} \frac{\partial \phi_{1}}{\partial Z_{0}} \\
& +\alpha \frac{\partial \phi_{1}}{\partial r} \frac{\partial \eta_{1}}{\partial Z_{0}} \frac{\partial \phi_{1}}{\partial Z_{0}}+\alpha \frac{\partial \phi_{0}}{\partial r} \frac{\partial \eta_{1}}{\partial Z_{0}} \frac{\partial \phi_{1}}{\partial Z_{1}}+\alpha \frac{\partial \phi_{1}}{\partial r} \frac{\partial \eta_{1}}{\partial Z_{0}} \frac{\partial \phi_{1}}{\partial Z_{0}} \\
& +\alpha\left(\frac{\partial \phi_{0}}{\partial r}\right)\left(\frac{\partial \eta_{1}}{\partial Z_{0}}\right)^{2}\left(\frac{\partial \phi_{1}}{\partial r}+\eta_{1} \frac{\partial^{2} \phi_{0}}{\partial r^{2}}\right) \\
& +\alpha \eta_{1} \frac{\partial \eta_{1}}{\partial Z_{0}}\left(\frac{\partial^{2} \phi_{0}}{\partial r^{2}} \cdot \frac{\partial \phi_{1}}{\partial Z_{0}}+\frac{\partial \phi_{0}}{\partial r} \frac{\partial^{2} \phi_{1}}{\partial r \partial Z_{0}}\right) \\
& +\alpha\left(\frac{\partial \phi_{0}}{\partial r}\right)^{2} \frac{\partial \eta_{1}}{\partial Z_{0}}\left(\frac{\partial \eta_{1}}{\partial Z_{1}}+\frac{\partial \eta_{2}}{\partial Z_{0}}\right)-\frac{\alpha}{2} \frac{\partial^{3}}{\partial r^{3}}\left(\frac{\partial \phi_{0}}{\partial r}\right)^{2}\left(\frac{\eta_{1}^{3}}{6}+\frac{\eta_{1} \eta_{2}}{3}\right),
\end{aligned}
$$


and

$$
\mathbf{O}\left[\eta_{3}, \phi_{3}\right]=-\eta_{2} \frac{\partial \phi_{1}}{\partial r}-\eta_{1} \frac{\partial \phi_{2}}{\partial r}-\frac{\eta_{1}^{2}}{2} \frac{\partial^{2} \phi_{1}}{\partial r^{2}}-\eta_{1} \eta_{2} \frac{\partial^{2} \phi_{0}}{\partial r^{2}}-\frac{\eta_{1}^{3}}{6} \frac{\partial^{3} \phi_{0}}{\partial r^{3}}
$$

\section{REFERENCES}

[1] Lord Rayleigh, The theory of sound, Vol. 2, Dover, 1945

[2] J. R. Melcher, Field coupled surface waves, M.I.T. Press, 1963

[3] D. H. Michael, Note on the electrostatic instability of a jet, Can. J. Phys. 45, 3179 (1967)

[4] M. C. Yuen, Nonlinear capillary instability of a liquid, J. Fluid Mech. 33, 151 (1968)

[5] A. H. Nayfeh, Nonlinear stability of a liquid jet, Phys. Fluids 13, 841 (1970)

[6] T. Kakutani, Y. Inoue and T. Kan, Nonlinear capillary waves on the surface of liquid column, J. Phy. Soc. Japan 37, 529 (1974)

[7] L. Crane, S. Birch and P. D. McCormack, The effect of mechanical vibrations on the break-up of a cylindrical n'ater jet in air, Brit. J. Appl. Phys. 15, 743 (1964)

[8] R. J. Donnelly and W. Glaberson, Experiments on the capillary instability of a liquid jet, Proc. Roy. Soc. (London) 290A, 547 (1966)

[9] K. C. Chaudhary and T. Maxworthy, The nonlinear capillary instability of a liquid jet. Part 2. Experiments on jet hehaviour before droplet formation, J. Fluid Mech. 96 part 2, 275 (1980)

[10] S. K. Malik and M. Singh, Nonlinear break-up of an electrohydrodynamic jet, Quart. Appl. Math. 41, No. 3, $273(1983)$

[11] J. R. Pierce, An interesting wave amplifier, IRE Transactions on Electron Devices, ED-7, 3 (1960)

[12] G. B. Whitman, Linear and nonlinear waves, John Wiley, New York, 1974

[13] V. I. Karpman, Nonlinear waves in dispersive media, Pergamon Press, 1975

[14] R. Kant, R. K. Jindia and S. K. Malik, Finite amplitude surface waves in electrohydrodynamics, Quart. Appl. Math. 39, No. 1, 23 (1981)

[15] V. E. Zakharov and A. B. Shabat, Exact theory of two-dimensional self-focusing and one-dimensional self-modulation of waves in nonlinear media, Soviet Phys., J.E.T.P. 34 (1972) 